\title{
Thermal conductivity and gap structure of the superconducting phases of $\mathrm{UPt}_{3}$
}

\author{
H. Suderow, J.P. Brison*, A. Huxley and J. Flouquet \\ Département de Recherche Fondamentale sur la Matière \\ Condensée, SPSMS,CEA/Grenoble, 17 rue des Martyrs, 38054 Grenoble cedex 9, France. \\ * Centre de Recherches sur les Très Basses Températures, CNRS, BP 166, 38042 Grenoble-Cedex \\ 9, France
}

(June 25, 2021)

\begin{abstract}
We present new measurements of the thermal conductivity $(\kappa)$ of $\mathrm{UPt}_{3}$ down to very low temperatures $(16 \mathrm{mK})$ and under magnetic fields (up to $4 \mathrm{~T}$ ) which cover all the superconducting phases of $\mathrm{UPt}_{3}$. The measurements in zero field are compared with recent theoretical predictions for the thermal conductivity, which is dominated by impurity states at the lowest temperatures studied. The measurements under magnetic field at low temperatures are surprising since they don't show the expected low field square root dependence, $\kappa \propto \sqrt{B}$. The discontinuity of $d \kappa / d T$ at $\mathrm{T}_{c}$ changes drastically when passing from the high field low temperature $\mathrm{C}$ phase to the low field high temperature A phase : this is related to the change of the symmetry of the superconducting order parameter when crossing the $\mathrm{A} \rightarrow \mathrm{C}$ phase transition.
\end{abstract}

To be published in Journal of Low Temperature Physics 


\section{INTRODUCTION}

$\mathrm{UPt}_{3}$ is widely believed to be an unconventional superconductor. The existence of several different superconducting phases in the B- P-T phase diagramm is experimentally well confirmed, and can only be explained by a change of the symmetry of the order parameter (OP) when passing from one superconducting phase to another (for reviews, see e.g. Ref. [1]). This, in turn, can only be understood if additional symmetries, other than gauge symmetry, are broken at the superconducting transition. The hexagonal crystal structure of this compound restricts the possible OP to 8 one-dimensional (so called A,B) and 4 twodimensional (E) irreducible representations of the crystal point group, which are further classified according to the parity of the superconducting wave functions (index $\mathrm{u}$ for odd and $g$ for even parity) [目]. In some of the irreducible representations the order parameter changes sign under some of the point group symmetries, implying points or lines of zeros of the gap on the Fermi surface. Much theoretical and experimental work has been done in order to try to find the symmetry of the OP in this compound, but up to now, no definite consensus emerges. The thermal conductivity is sensitive to the position and type of the nodes (line or point nodes) of the superconducting gap, which itself depends on the symmetry of the OP. Previous work (See Refs. [2,3,5, 8] for experimental and Refs. [9] 19] for theoretical work) has already demonstrated that the thermal conductivity $(\kappa)$ in this compound is of electronic origin at very low temperature, and thus sensitive to the electronic excitations in the superconducting state. Compared to the specific heat $\mathrm{C}, \kappa$ is a directional probe, and it seems [5,6] that the anomaly present in the specific heat below $100 \mathrm{mK}$ does not influence $\kappa$. Thus, a directional study of $\kappa$ down to very low temperatures is expected to give much information about the gap structure of $\mathrm{UPt}_{3}$. Note that the first measurements [3.5] (8) had already stressed the necessity of choosing a model for the OP yielding a hybrid gap with a line node in the basal plane and point node along the c axis. Measurements on far improved samples to lower temperatures are now expected to distinguish between such models. The discussion of our zero field data will be limited, as in all recent theoretical 
[15 19] and experimental [5 8] work, to two plausible symmetries for the OP that appear consistent with the observed properties of superconductivity in $\mathrm{UPt}_{3}$ : the $\mathrm{E}_{1 g}$ and $\mathrm{E}_{2 u}$ representations of the order parameter which both lead to a hybrid gap, with a line node in the basal plane and nodes along the c axis where the gap vanishes respectively linearly $\left(\mathrm{E}_{1 g}\right)$ or quadratically $\left(\mathrm{E}_{2 u}\right)$ with the polar angle $\theta$. Up to now, no experimental distinction could be made between the two models on the basis of the thermal conductivity. Since the work of K. Behnia et al. [3] measurements (Lussier et al. [7] for $\mathrm{T} \geq 100 \mathrm{mK}$ ) emphasizing the appearance of an anisotropy of the thermal conductivity below $\mathrm{T}_{c}$ concluded that the gap was of the $\mathrm{E}_{1 g}$ type, and further measurements by the same group to lower temperatures $(\mathrm{T} \geq 50 \mathrm{mK})[8]$ were interpreted to favour $\mathrm{E}_{2 u}$. We [5] measured the thermal conductivity down to $35 \mathrm{mK}$, and showed that although some features of our data, like the power law behaviours, seemed to favour the $\mathrm{E}_{2 u}$ model, others did not, like the temperature dependence of the anisotropy. In fact it was shown [15 19] on the basis of the data of Lussier et al. [8] that the existing experimental data could not differentiate between the two models. An important point is that for the lowest temperatures, which should be best suited to distinguish between the two gap structures, theory predicts that the pair breaking effect of impurities and defects produces a band of impurity bound states which restores a behaviour of the thermal conductivity comparable to that of the normal state. This regime was never observed up to now, as the measurements were not extended to low enough values of $\mathrm{T} / \mathrm{T}_{c}$. Our data down to $16 \mathrm{mK}$ show the onset of this type of behaviour, which is accompanied by drastic changes on the anisotropy of $\kappa$ below $30 \mathrm{mK}$. In addition, the experimental resolution has been considerably improved, thus our data give a much better basis for a more detailed discussion of the thermal conductivity. We discuss extensively our zero field data, and also present new measurements under magnetic fields. Measurements under field in the mixed state give an additional means of probing the gap structure [3, 24 26]. Indeed, the possibility of a hybrid gap such as that in the $\mathrm{E}_{1 g}$ and $\mathrm{E}_{2 u}$ models was proposed by Yin and Maki [25] on the basis of the mixed state thermal conductivity data of Behnia et al. [3: Our data are more systematic and show new features of $\kappa$ in the mixed state, arising both 
at very low temperatures and near the tetracritical point, where the three superconducting phases of $\mathrm{UPt}_{3}$ coalesce.

\section{EXPERIMENTAL}

The crystals used for the measurements were cut at adjacent sites of the same mother crystal and followed exactly the same heat treatment. Their critical temperature $\mathrm{T}_{c}$ is the same, their specific heat shows [5] clearly the double superconducting transition and their residual resistivities are among the lowest ever reported $\left(\rho_{0}=0.54 \mu \Omega \mathrm{cm}\right.$ for the current $\mathrm{j} / / \mathrm{b}$, and $\rho_{0}=0.17 \mu \Omega \mathrm{cm}$ for $\mathrm{j} / / \mathrm{c}$ [5]). The samples were heated by a PtW alloy strain gauge heater, and the temperature was measured by two matched Matshushita $68 \Omega$ carbon thermometers. The accuracy of the measurements has been significantly improved. We could achieve a $1 \%$ resolution at $500 \mathrm{mK}, 3 \%$ at $50 \mathrm{mK}$ and $10 \%$ at $16 \mathrm{mK}$ for the measured anisotropy $\kappa_{c} / \kappa_{b}$. By comparison, the authors of Ref. [8] give error bars of $1 \%$ at $150 \mathrm{mK}$ and $5 \%$ at $50 \mathrm{mK}$ on $\kappa$, whereas for the anisotropy $\kappa_{c} / \kappa_{b}$, the error bars are estimated to be $15 \%$ above $50 \mathrm{mK}$ [18]. We improved our experimental method (described in Ref. [5]) in the following points: (i) better electronics, and extensive time averaging to reduce noise. As a consequence, a continous run from $16 \mathrm{mK}$ to $1 \mathrm{~K}$ fully computer controlled took 3 days of measuring time. (ii) The long time averaging was possible due to the very high temperature stability of our dilution refrigerator, and its low temperature minimum : $5 \mathrm{mK}$. (iii) In this dilution refrigerator we could calibrate our thermometers during the same experiment against a Ge thermometer, a paramagnetic CMN salt and a ${ }^{3} \mathrm{He}$ vapor pressure thermometer. The lowest measuring temperature for our samples was limited to $15 \mathrm{mK}$ by their self heating due to the $\mathrm{U}$ radioactivity. The problem of the magnetoresistance of the thermometers (the improved sensitivity is payed for by important changes of the calibration under magnetic field) was solved by making a new calibration against a standard Ge thermometer in the zero field compensated region of the magnet at every field. The experimental error bars become

more important when increasing the magnetic field, and will be shown in the figures when 
necessary. In high magnetic fields (3T), and for $\mathrm{j} / / \mathrm{c}$, the thermal conductivity of $\mathrm{UPt}_{3}$ is extremely large : 3 orders of magnitude larger than at $16 \mathrm{mK}$ and zero field, due to the low value of the residual resistivity $\rho_{0}$ for $\mathrm{j} / / \mathrm{c}\left(\rho_{0}=0.17 \mu \Omega \mathrm{cm}\right)$. This, and the poor geometric factor of our sample for $\mathrm{j} / / \mathrm{c}$ (roughly $2 \mathrm{~mm}$ length, compared with $6 \mathrm{~mm}$ for the sample measured for $\mathrm{j} / / \mathrm{b}$ ) conspired to make the measurements at $3 \mathrm{~T}$ and very low temperatures extremely difficult. Therefore, at $3 \mathrm{~T}$ and for $\mathrm{j} / / \mathrm{c}$ we only measured above $100 \mathrm{mK}$. Taking another sample with better geometry would have solved this problem, but our interest was focused on the superconducting phase of $\mathrm{UPt}_{3}$. More than $60 \kappa(\mathrm{T})$ runs under different magnetic fields were done in order to scan the B-T phase diagramm. All magnetic fields where applied along the same axis as the heat current $(B / / j / / b$ and $B / / j / / c)$.

\section{NORMAL PHASE}

The normal phase behaviour of the thermal conductivity of $\mathrm{UPt}_{3}$ shows some features important for the understanding of the superconducting phase. In Figure 1 we have plotted $\kappa(T)$ for $\mathrm{j} / / \mathrm{b}$ and $\mathrm{j} / / \mathrm{c}$, at $0 \mathrm{~T}$ and at $3 \mathrm{~T}$ where the normal phase is recovered. The dominant scattering mechanisms of the electrons are the same as in the electrical conductivity : (i) elastic scattering of electrons by impurities and (ii) inelastic scattering between electrons. The first scattering mechanism (i) leads to a linear temperature dependence of the thermal conductivity and is related to the temperature independent part of the resistivity by the Wiedemann-Franz law : $\kappa / T=L_{0} / \rho_{0}\left(L_{0}=2.4410^{-8} W / K^{2} \mathrm{~cm}\right)$. As seen in Fig. 1 this mechanism dominates at very low temperatures $\mathrm{T} \leq 100 \mathrm{mK}$, below which $\kappa / T$ only varies by $10 \%$ as $\mathrm{T} \rightarrow 0 \mathrm{~K}$. The ratio of the thermal conductivities extrapolated down to $\mathrm{T}=0 \mathrm{~K}$ along the $\mathrm{b}$ and the $\mathrm{c}$ axis is 3 . This ratio is usually said [7,8,15] 18 to be related to the anisotropy of the Fermi velocities, rather than to the anisotropy of the scattering rate $\tau$, which is assumed to be isotropic. Note that this point is not experimentally demonstrated. Nevertheless we will also take $\tau$ to be isotropic, in order to compare our data with theory.

The second mechanism (ii) leads to the well known Fermi liquid $\mathrm{T}^{2}$ behaviour of the electrical 
resisitivity and is of growing importance with increasing temperature. Deviations from the simple Wiedemann-Franz law are then expected : one expects again $\kappa / T=L /\left(\rho_{0}+A T^{2}\right)$ but now with a temperature dependent Lorentz number $\mathrm{L}(\mathrm{T}) \leq L_{0}$.

In order to verify this point, we also measured the electrical resistivity of our samples at 3 T. In high purity samples, $\omega_{c} \tau$ ( $\omega_{c}$ being the cyclotron frequency) becomes larger than 1 at low temperatures. This complicates the temperature dependence of the resistivity at $3 \mathrm{~T}$, so that we did not get the expected simple Fermi liquid behaviour $\rho=\rho_{0}+A T^{2}$. Such effects have been already observed in $\mathrm{UPt}_{3}[21,22$. Nevertheless we can roughly estimate that the Lorentz number varies from $0.95 \mathrm{~L}_{0}$ at $\mathrm{T}=0 \mathrm{~K}$ to $0.8 \mathrm{~L}_{0}$ at $\mathrm{T}=600 \mathrm{mK}$. We note that the positive magnetoresistance of $\mathrm{UPt}_{3}\left(\rho_{0}\right.$ varies for $\mathrm{j} / / \mathrm{b}(\mathrm{j} / / \mathrm{c})$ by roughly $20 \%(10 \%)$ between

0 and $3 \mathrm{~T}$ [3.22 ) leads one to expect that $\kappa$ decreases with $\mathrm{B}$, as was already observed by Behnia et al. [3] but contrary to what was shown by Lussier et al. [8], although these authors might have corrected qualitatively for the magnetoresistance, assuming the validity of the Wiedemann-Franz law in all the measured temperature range.

\section{SUPERCONDUCTING PHASE}

The thermal conductivity in zero field is shown in Fig. 1. No significant change is observed at $\mathrm{T}_{c}$. It is important to realize that up to now quantitative comparison with theory in the superconducting phase can only be done easily when elastic scattering with impurities prevails. As shown in Fig. 1, $\kappa$ at $\mathrm{T}_{c}$ is roughly half the value of the zero temperature extrapolation of $\kappa / T$ in the normal state. This means that at $\mathrm{T}_{c}$ both mechanisms (i) and (ii) are of equal importance. When entering the superconducting state, $\kappa$ rapidly drops as the superconducting gap opens over the Fermi surface. Therefore, the inelastic scattering rate in the superconducting state should diminish more rapidly than in the normal state. A qualitative treatment of the inelastic scattering rate has been proposed by Fledderjohann and Hirschfeld [17], extrapolating the normal phase scattering rate taken to be proportional to $1 /\left(\rho_{0}+A T^{2}\right)$. Norman and Hirschfeld [18] also made an extensive analysis of Fermi surface 
effects on $\kappa$, including inelastic scattering, and demonstrated the difficulty of fitting $\kappa$ in this temperature range. As suggested in Ref. [17], they tried to fit at the same time $\kappa(T)$ and the anisotropy $\kappa_{c} / \kappa_{b}$, shown in Fig. 2. This quantity has little temperature dependence in the normal phase. As shown in the inset of Fig. 2, our enhanced resolution permits us to observe a kink in $\kappa_{c} / \kappa_{b}$ against temperature at roughly $550 \mathrm{mK}$, which should correspond to the onset of superconductivity. Near $\mathrm{T}_{c}$ (for $\left.\mathrm{T} \leq \mathrm{T}_{c}\right), \kappa_{c} / \kappa_{b}$ is independent of temperature, as shown in Ref. [5], but contrary to what was observed by Lussier et al. [7, 8]. Below 0.8T $\mathrm{T}_{c}^{-}$ $\left(\mathrm{T}_{c}^{-}=480 \mathrm{mK}\right) \kappa_{c} / \kappa_{b}$ starts to decrease and seems to tend towards a finite zero temperature extrapolation. However below $30 \mathrm{mK}\left(0.07 \mathrm{~T}_{c}^{-}\right)$a stronger dependence on temperature sets in. Norman and Hirschfeld calculated $\kappa_{c} / \kappa_{b}$ using many different functions to describe the $\mathrm{OP}$ within the $\mathrm{E}_{1 g}$ and $\mathrm{E}_{2 u}$ irreducible representations, and one of the curves they calculate (Fig. 8 of Ref. [18]) fits our measurement of the anisotropy $\kappa_{c} / \kappa_{b}$ well, however their calculated temperature dependence of $\kappa_{b}$ and $\kappa_{c}$ do not correspond with our data. Nevertheless, we note that a temperature independent anisotropy $\kappa_{c} / \kappa_{b}$ near $\mathrm{T}_{c}$ seems to be only reproduced within $\mathrm{E}_{2 u}$ in their calculations. At the lowest temperatures $(\mathrm{T} \rightarrow 0 \mathrm{~K})$ the thermal excitations which drive the heat current have $\vec{k}$ vectors corresponding only to the Fermi surface regions near the nodes of the gap, and inelastic scattering can be ignored, so that the interpretation of $\kappa$ is expected to be simpler. We now discuss impurity effects which turn out to be important in this limit.

In non conventional superconductors with nodes of the gap, the pair breaking effect of even non magnetic impurities or defects leads to a finite density of states at the Fermi level, due to virtual bound states forming a band of width $\gamma$ (see Refs. [9 11, 13 [18, 31, 34]). This band is easier to observe when the impurities are in the unitarity scattering limit (scattering phase shift $\delta=\pi / 2$ ), and in heavy fermion superconductors, impurity scattering is expected to be in this limit 9]. Thus, in $\mathrm{UPt}_{3}$, the thermodynamics is expected to be governed by the propeties of this band for $\mathrm{T} \leq \gamma$.

Before discussing the peculiar properties of this band which leads (see below) to a finite $\kappa / T$ for $\mathrm{T} \rightarrow 0 \mathrm{~K}$, we focus on the power law behaviour expected for intermediate tempera- 
tures: $\gamma \leq T \ll T_{c}$. In this temperature region, the impurity induced band can be neglected, as well as inelastic scattering effects. In the case of resonant scattering of the thermal excitations by impurities, simple power laws are expected, controled by the gap nodes. Our measurements of $\kappa / T$ below $80 \mathrm{mK}$ are shown in Fig. 3 as a function of $\mathrm{T}^{2}$. We can observe two regimes : (i) between $80 \mathrm{mK}$ and $30 \mathrm{mK}$ a $\mathrm{T}^{3}$ power law behaviour of $\kappa$ for both axis, as expected for a hybrid gap with resonant scattering and (ii) below $30 \mathrm{mK}$ the effects of the impurity induced band of quasiparticles leading to a finite value of $\kappa / T$ for $\mathrm{T} \rightarrow 0 \mathrm{~K}$. Note that the extrapolation of the data for $\mathrm{T} \geq 30 \mathrm{mK}$ gives a negative zero temperature extrapolation for $\mathrm{j} / / \mathrm{c}$ (zero for $\mathrm{j} / / \mathrm{b}$ ), which implies that the anisotropy $\kappa_{c} / \kappa_{b}$ still depends on temperature down to $30 \mathrm{mK}$ (see Fig. 2). In an earlier paper [5] we argued that observing the same power laws for both axis is in favour of the $\mathrm{E}_{2 u}$ model, as the quadratic angular dependence of the nodes near the $\mathrm{c}$ axis leads to a high density of thermal excitations comparable to the density in the basal plane [17]. Nevertheless, the theoretical analysis of the authors of Refs. [15, 16, 18, 19] demonstrated that this observation could also be consistent with an $\mathrm{E}_{1 g}$ state. Barash and Svidzinsky [19] examined the expected power laws in detail, and they find to leading order in $\mathrm{T}$ :

- for $\mathrm{j} / / \mathrm{b}$ (heat current in the basal plane ): $\kappa_{b}=T^{3}\left(a_{b}+b_{b} \ln ^{2}\left(T / \Delta_{0}\right)+c_{b} \ln \left(T / \Delta_{0}\right)\right)$ for $\mathrm{E}_{1 g}$ and $\mathrm{E}_{2 u}$;

- for $\mathrm{j} / / \mathrm{c}$ (heat current perpendicular to the basal plane): $\kappa_{c}=T^{3}\left(a_{c}+b_{c} l^{2}\left(T / \Delta_{0}\right)+\right.$ $\left.c_{c} \ln \left(T / \Delta_{0}\right)\right)$ for $\mathrm{E}_{2 u}$ and $\kappa_{c}=a_{c} T^{3}$ for $\mathrm{E}_{1 g}$;

with coefficients $a, b$ and $c$ which depend on the parametrization of the gap near the nodes, and even on contributions of the order parameter from regions of the Fermi surface far from the nodes [19]. The power laws expected on $\kappa_{c}$ for $\mathrm{E}_{1 g}$ and $\mathrm{E}_{2 u}$ differ mainly by a logarithmic factor $\left(1+\frac{b_{c}}{a_{c}} l^{2}\left(T / \Delta_{0}\right)+\frac{c_{c}}{a_{c}} \ln \left(T / \Delta_{0}\right)\right)$. As seen in Figure 3, both $\kappa_{b}$ and $\kappa_{c}$ do not follow a pure power law, but need a negative zero temperature extrapolation to be fitted. The formulas given above (for $\mathrm{E}_{2 u}$ ) fit our data well, but with negative coefficients $\mathrm{b}_{b}$ and $\mathrm{b}_{c}$, which may not have a clear physical meaning. Therefore, on the basis of power law behaviours we are not able to discriminate unambiguously between $\mathrm{E}_{1 g}$ and $\mathrm{E}_{2 u}$. 
The lowest temperature regime $(\mathrm{T} \leq \gamma)$ might still be used for the identification of the order parameter. Indeed theoretical work has shown [15, 16, 18] that the existence of the impurity induced band of quasiparticles does not wash out completely the sensitivity of the thermal conductivity to the choice of the OP. We will follow closely the discussion of Graf et al. [16]. They predict for $T \leq \gamma: \kappa_{i} / T=\alpha_{i}+\beta_{i} T^{2}$ with $\alpha_{i}$ and $\beta_{i}$ which depend on the choice of the OP and of the direction of the heat current :

- For $\mathrm{i}=\mathrm{b}$ (heat current in the basal plane) $\kappa_{b}$ is the same for $\mathrm{E}_{1 g}$ and $\mathrm{E}_{2 u}$, as both predict a line node in the basal plane. Moreover, $\alpha_{b}$ is universal, i.e. independent of the impurity concentration $n_{i}$ although it depends on the form of the gap near the node, and $\beta_{b}=\frac{7 \pi^{2} k_{B}^{2}}{60 \gamma^{2}} \alpha_{b}$.

- For $\mathrm{i}=\mathrm{c}, \alpha_{c} \propto \gamma$ and $\beta_{c}=2.5 \frac{\beta_{b}}{\alpha_{b}} \alpha_{c}$ for $\mathrm{E}_{1 g}$, but for $\mathrm{E}_{2 u}, \alpha_{c} \propto \alpha_{b}$ is universal and $\beta_{c}=\frac{\beta_{b}}{\alpha_{b}} \alpha_{c}$.

The inset of Fig. 3 shows $\kappa / T$ as a function of $\mathrm{T}^{2}$ at the lowest temperatures $(\mathrm{T} \leq 35$ $\mathrm{mK}$ ) together with the fits to the predicted formulas. For the thermal conductivity in the basal plane, the fit gives $\alpha_{b}=0.18 \mathrm{~mW} / \mathrm{K}^{2} \mathrm{~cm}$, of the same order of magnitude as $\alpha_{b} \approx 1 \mathrm{~mW} /\left(K^{2} \mathrm{~cm}\right)$ estimated in Ref. [15]. It also yields $\beta_{b} / \alpha_{b}=4.210^{3} / K^{2}$, therefore $\gamma \approx 17 \mathrm{mK}$. Note that with a less pure sample we should have been able to observe the expected law over a larger range of temperatures. Assuming that the impurity concentration $\mathrm{n}_{i}$ is the same for both measured crystals, and having fixed $\alpha_{b}$ and $\beta_{b} / \alpha_{b}$ only $\alpha_{c}$ can be varied as a free parameter in order to reproduce the measured curve for $\mathrm{j} / / \mathrm{c}$ [15]. For $\mathrm{E}_{2 u}$ the best agreement is obtained with $\alpha_{c}=0.2 \mathrm{~mW} / \mathrm{K}^{2} \mathrm{~cm}$ and for $\mathrm{E}_{1 g}$ with $\alpha_{c}=0.11 \mathrm{~mW} / \mathrm{K}^{2} \mathrm{~cm}$. As shown in Fig. 3, the fit to $\mathrm{E}_{1 g}$ is better, but only in a small temperature range $(16 m K \leq T \leq$ $25 m K)$. Nevertheless, the assumption that $\gamma$ is the same for both measured samples may not be justified. We recall that the assumption of an isotropic scattering rate may not hold, and that even if both samples come from the same mother crystal and had exactly the same heat treatment, differences in the impurity (or defect) concentration can never be excluded. Indeed, although the onset of a finite resistivity occurs at the same temperature for both samples, the width of the superconducting transition measured by resistivity is $17 \mathrm{mK}$ for 
$\mathrm{j} / / \mathrm{b}$, but only $8 \mathrm{mK}$ for $\mathrm{j} / / \mathrm{c}$ (10\%-90\% criterion). The broadening of the superconducting transition could be correlated to the sensitivity to stress produced in the cutting of the material despite the subsequent annealing. Note that our measurements under magnetic fields show a clear anomaly in $\kappa$ at $\mathrm{T}_{c}$. As shown in the inset of Fig. 4 this anomaly coincides with the onset of a finite resistivity, thus with the lowest part of the resistive transition. Therefore, differences in the transition width, although they show that the samples are different, do not give much information about the defect concentration in the bulk of the material. We note that in order to explain our data within $\mathrm{E}_{2 u}$, the impurity concentration $\mathrm{n}_{i} \propto \gamma^{2}$ 15 should be 2.5 times lower for $\mathrm{j} / / \mathrm{c}$ than for $\mathrm{j} / / \mathrm{b}$. One way of solving this problem is to measure less pure samples (in order to observe the expected laws in a larger range of temperatures), again for both axis and down to the lowest possible temperatures. This will also give an experimental test of the prediction of a universal value of $\kappa / T(T \rightarrow 0 K)$ for the heat current in the basal plane. Note that the upper critical field anisotropy $\left(\mathrm{B}_{c 2}\right.$ is slightly Pauli limited for $\mathrm{B} / / \mathrm{c}$ but not for $\left.\mathrm{B} \perp \mathrm{c}\right)$ is an important argument in favour of an odd parity OP in $\mathrm{UPt}_{3}$ (see Ref. [23]). It will be very difficult to explain $\mathrm{B}_{c 2}$ within $\mathrm{E}_{1 g}$.

One important point not understood at present is the discrepancy between the value of $\gamma$ estimated from the normal phase data (with the impurity scattering rate $\Gamma_{0} / T_{c} \approx 510^{-2}$ we obtain $\gamma \approx 50 \mathrm{mK}$; see Ref. [15] for the formulas), which is 3 times larger than the one found from our low temperature fit. Indeed, as pointed out by Norman and Hirschfeld [18] the data at higher temperatures $\left(\gamma \ll T \leq T_{c}\right)$ seem to be difficult to fit with such a low impurity scattering rate. This may suggest that the description of the impurities and defects in $\mathrm{UPt}_{3}$ only in terms of s-wave scattering in the unitarity limit is too simplified for the discussion of subtle very low temperature effects. It also stresses the lack of experimental knowledge on the nature of the dominant scattering centers in this compound. 


\section{MIXED PHASE}

Figure 4 shows an example for our temperature scans at different magnetic fields. The first striking feature is that under magnetic fields an anomaly appears, which was not visible in zero field. More precisely, there is a jump in the derivative of $\kappa: \mathrm{d} \kappa / \mathrm{dT}$ at $\mathrm{T}_{c}$. This is predicted to happen already in conventional s-wave superconductors, and the interesting point is that, from the superconducting side, $\mathrm{d} \kappa / \mathrm{dT}$ is related to the order parameter. At intermediate temperatures $\left(100 \mathrm{mK} \leq \mathrm{T} \leq \mathrm{T}_{c}\right), \kappa$ first decreases at low fields as a function of $\mathrm{B}$, and then increases up to its value in the normal phase (this is seen more clearly in the Fig. 8, where we plot $\kappa$ as a function of B). At very low temperatures $(\mathrm{T} \leq 50 \mathrm{mK})$, no decrease at low fields is observed and $\kappa$ increases continuously with B. Qualitatively speaking, there are two competing effects : an enhancement of the thermal conductivity with the magnetic field towards the normal phase value, and the scattering of the thermal excitations present outside the vortex cores by the vortices which may tend to diminish $\kappa$ (compared to the zero field value). The understanding of the thermal conductivity of clean superconductors in the mixed state is difficult due to the complex interplay between these

effects, and new theoretical calculations are needed in order to understand $\kappa(\mathrm{B})$. Nevertheless some conclusions can be drawn.

All of these effects strongly depend on the relative direction of the magnetic field and the heat current. Our choice was to measure $\kappa$ with the field always parallel to the heat current, along the $\mathrm{c}$ axis and in the basal plane, in order to make the interpretation easier. Also, the magnetic field was always changed in the normal phase (field cooled). We checked that only a small irreversibility exists at very low fields $(\mathrm{B} \leq 0.03 \mathrm{~T})$.

Before discussing the data, we note that we recover the phase diagram of $\mathrm{UPt}_{3}$ when plotting $\mathrm{T}_{c}$ obtained by the anomaly of $\kappa$ as a function of the magnetic field (see Fig. 5). The $\mathrm{B} \rightarrow \mathrm{C}$ line could also be observed due to anomalies shown in Fig. 8 on the field dependence of $\kappa$, but no clear signature could be found of the $\mathrm{A} \rightarrow \mathrm{B}$ phase transition. We will first discuss the thermal conductivity near $\mathrm{T}_{c}(\mathrm{~B})$, then focus on $\kappa(B)$ as $\mathrm{T} \rightarrow 0 \mathrm{~K}$, and finally 
discuss $\kappa(B)$ at intermediate temperatures.

In the literature (see Refs. [3, 4, 25, 26] for the discussion of $\mathrm{UPt}_{3}$ ), it is generally the jump in $d \kappa / d B$ which is discussed. We have measured $d \kappa / d T$ because our resolution for temperature scans was much better than for field scans, but the main conclusions should remain the same. In Fig. 6 we show the measured $d \kappa / d T$ at $\mathrm{T}_{c}$ as a function of the magnetic field, normalized to the normal state values : $\left(d \kappa_{s} / d T-d \kappa_{n} / d T\right) /\left(\kappa_{n} / T_{c}(B)\right)$, where the subscript $\mathrm{s}$ means approaching $\mathrm{T}_{c}$ from the superconducting state, and $\mathrm{n}$ from the normal state. As already mentioned [25,26] and shown by the measurements of Behnia et al. [3,4] in $\mathrm{UPt}_{3}$, the slope $d \kappa_{s} / d B$, and therefore also $\mathrm{d} \kappa_{s} / \mathrm{dT}$, depends on the direction of current and field, and is related to the gap structure. Therefore, if the topology of the gap changes when passing from the high temperature low field A phase to the low temperature high field $\mathrm{C}$ phase, a jump of $d \kappa / d T$ should be observed at the $\mathrm{A} \rightarrow \mathrm{C}$ phase transition. Indeed, as shown in Fig. 6, we clearly observe this jump for both orientations of the field at the $\mathrm{A} \rightarrow \mathrm{C}$ phase transition (0.5 $\mathrm{T}$ for $\mathrm{B} / / \mathrm{b}$ and $1 \mathrm{~T}$ for $\mathrm{B} / / \mathrm{c})$. If no phase transition took place, the overall behaviour of the measured quantity would be parabolic like, with $\left(d \kappa_{s} / d T-d \kappa_{n} / d T\right) /\left(\kappa_{n} / T_{c}(B)\right)=0$ at $\mathrm{B}=0$ and at $B=B_{c 2}(T=0)$, with a maximum at an intermediate field. We expect this parabola to depend on the topology of the superconducting gap. So it might be possible to draw more conclusions about the gap structure of the $\mathrm{A}$ and the $\mathrm{C}$ phases, from which little is known, by a careful theoretical analysis of these data.

At very low temperatures $(\mathrm{T} \leq 50 \mathrm{mK})$ we observe a continuous increase (Fig. 7$)$ of $\kappa(B)$ up to its normal phase behaviour, which we will discuss now. First, we recall the situation in clean s-wave superconductors. Within a naive model, one might think that in the limit $\mathrm{T} \rightarrow 0 \mathrm{~K}$ the contribution to the thermal conductivity coming from the electronic excitations within the vortex cores would dominate $\kappa$, as this type of excitation dominates the density of states (in s-wave superconductors). One would then expect that $\kappa$ scales roughly as $\frac{B}{B_{c 2}} \kappa_{n}$, but this turns out not to be correct. Indeed, the low group velocity of the excitations within the vortex cores leads to a small contribution of this type of excitations to the thermal 
conductivity. Vinen et al. 28 estimate $\kappa_{\text {vortex cores }} \approx 10^{-2} \frac{B}{B_{c 2}} \kappa_{n}$ for pure $\mathrm{Nb}$. We observe in $\mathrm{UPt}_{3}$ an increase of $\kappa$ with the magnetic field which scales roughly as $\frac{B}{B_{c 2}} \kappa_{n}$ for $\mathrm{B} / / \mathrm{j} / / \mathrm{b}$ and as $\frac{1}{3} \frac{B}{B_{c 2}} \kappa_{n}$ for $\mathrm{B} / / \mathrm{j} / / \mathrm{c}$ at low fields (Fig. 7). This is a surprising result which is not explained with the existing models. It might be related to the peculiar magnetic field behaviour of the density of states of unconventional superconductors with a line of nodes of the gap. Indeed, it has been predicted that in such a case, the density of states would be dominated by the contribution of the thermal excitations of the superconducting phase outside the vortex cores, leading to $\mathrm{N}\left(\mathrm{E}_{F}\right) \propto \sqrt{B}$ instead of $\mathrm{N}\left(\mathrm{E}_{F}\right) \propto B$ when the main excitations are those in the vortex cores. Note that this is valid at low fields for $B_{c 1}<B \ll B_{c 2}$ [30,19]. Again, in a naive model, one would expect that $\frac{\kappa}{T}(\mathrm{~B})$ as $\mathrm{T} \rightarrow 0 \mathrm{~K}$ follows the density of states, but no signature of the expected square root behaviour due to the line of nodes predicted within $\mathrm{E}_{1 g}$ and $\mathrm{E}_{2 u}$ is found in our data. Nevertheless, it is already known that in the case of clean s-wave superconductors, the thermal conductivity does not in general follow the density of states. We note also that the thermal excitations outside the vortex cores are scattered by the vortices (see below), and this may considerably influence $\frac{\kappa}{T}(\mathrm{~B})$ also in the limit $\mathrm{T} \rightarrow 0 \mathrm{~K}$. A rough square root behaviour was found in the specific heat $\mathrm{C}$ by Ramirez et al. [29], although at suspiciously high temperatures $\left(150 \mathrm{mK} ; \mathrm{T}_{c} / 3\right)$, and large fields (up to $\left.\mathrm{B}_{c 2} / 3\right)$. These measurements are therefore influenced by the field dependence of $\mathrm{T}_{c}$ and by a huge low temperature anomaly present in $\mathrm{C}[37,38]$. The origin of this anomaly, which appears already at $100 \mathrm{mK}$ in zero field is not understood at present, but it is for sure not connected with the superconducting state [38]. So the measurements of Ramirez et al. 29] are clearly not simply related with the field dependence of the density of states at $\mathrm{T} \rightarrow 0 \mathrm{~K}$. The advantage of the thermal conductivity is that it seems not to be influenced by this anomaly. Its origin may therefore be related to localized modes which do not carry heat [5]. In summary, it is clear that $\kappa(B)$ as $\mathrm{T} \rightarrow 0 \mathrm{~K}$ has a peculiar behaviour in $\mathrm{UPt}_{3}$ not explained by the existing models. One must explain not only the linear behaviour of $\kappa$ with $\mathrm{B}$ at low fields, but also the anisotropy found for the constants of proportionality $\left(\kappa \simeq B / B_{c 2}\right.$ for 
$\mathrm{B} / / \mathrm{j} / / \mathrm{b}$ and $\kappa \simeq \frac{1}{3} B / B_{c 2}$ for $\left.\mathrm{B} / / \mathrm{j} / / \mathrm{c}\right)$.

As $\kappa(B)$ scales roughly as $\frac{1}{3} \frac{B}{B_{c 2}} \kappa_{n}$ for $\mathrm{B} / / \mathrm{j} / / \mathrm{c}, \kappa(B)$ cannot remain linear in $\mathrm{B}$ up to $\mathrm{B}_{c 2}$. Indeed, at roughly $0.8 \mathrm{~B}_{c 2}, \kappa_{c}$ strongly deviates from the linear behaviour in order to reach the normal state value at $\mathrm{B}_{c 2}$, whereas $\kappa_{b}$ is roughly linear in the whole field range up to $\mathrm{B}_{c 2}$. This behaviour is observed for the extrapolation $\mathrm{T} \rightarrow 0 \mathrm{~K}$, as well as at $100 \mathrm{mK}$, as shown in Fig. 8. Note that Behnia et al. [3] already observed a large value for $\mathrm{d} \kappa / \mathrm{dB}$ with $\mathrm{B} / / \mathrm{c}$ when the upper critical field is Pauli limited along the c axis [23]. This may influence $\kappa(\mathrm{B})$ near $\mathrm{B}_{c 2}$. This measurement, and the ones of Behnia et al. (Refs. [3, [1] in $\mathrm{UPt}_{3}$ and Ref. [24] in $\mathrm{URu}_{2} \mathrm{Si}_{2}$, another heavy fermion superconductor with a Pauli limited upper critical field along one crystallographic axis) are to our knowledge the only existing thermal conductivity measurements in the mixed phase of superconductors with a Pauli limited upper critical field. We could not find theoretical predictions in this case for $\kappa(B)$ near $\mathrm{B}_{c 2}$.

We now focus on the magnetic field behaviour of $\kappa$ between $100 \mathrm{mK}$ and $\mathrm{T}_{c}$, shown in Fig. 8 and obtained by plotting our temperature scans as a function of B. As pointed out above, the scattering of thermal excitations by vortices leads to a diminution of $\kappa(B)$ visible at low fields. This type of scattering was indeed reported experimentally in s- wave superconductors (see e.g. Refs. [20,27]), and in $\mathrm{UPt}_{3}$ by Behnia et al. [3] and Huxley et al. [5]. In a simple model for low fields where vortices can be treated as independent scattering centers, the additional thermal resistivity $\mathrm{W}=1 / \kappa$ (Fig. 9) coming from scattering of the superconducting thermal excitations by the vortices is expected to be proportional to the magnetic field (see e.g. Refs. 20,27,36]). In s-wave superconductors, the law $\mathrm{W}=$ $\mathrm{W}(0)\left(1+\frac{\sigma l_{0}}{\Phi_{0}} B\right)\left(l_{0}\right.$ is the mean free path in the superconducting phase, $\Phi_{0}$ the flux quantum, and $\sigma$ the effective scattering width of the vortices) is observed at low fields and in a large range of temperatures. Our data do not show such a behaviour. To understand this, the field dependence of $\kappa$ for $\mathrm{T} \rightarrow 0 \mathrm{~K}$ has first to be explained. The fact that we do not observe the classical behaviour may indeed be related to the unconventional nature of superconductivity in $\mathrm{UPt}_{3}$. 


\section{CONCLUSION}

We have shown that the thermal conductivity shows a rich behaviour in the different superconducting phases of $\mathrm{UPt}_{3}$. Experimentally, we could reach low enough temperatures to enter the regime where the thermal conductivity is governed by impurity bound states. It is unclear if it is now possible to differentiate between $\mathrm{E}_{1 g}$ or $\mathrm{E}_{2 u}$ on the basis of this data, we can however parametrize the gap nodes for both models. It appears that measurements on samples of different purity down to the same lowest temperatures would be very useful. First, they could test the validity of the theoretical models, predicting for $\mathrm{E}_{1 g}$ as well as for $\mathrm{E}_{2 u}$ a universal value of $\kappa / T$ for $\mathrm{T} \rightarrow 0 \mathrm{~K}$ in the basal plane. Second, they might distinguish between $\mathrm{E}_{1 g}$ and $\mathrm{E}_{2 u}$ : theory predicts for the heat current along the c axis a universal value of $\kappa / T$ for $\mathrm{T} \rightarrow 0 \mathrm{~K}$ in the case of $\mathrm{E}_{2 u}$, but a sample dependent value for $\mathrm{E}_{1 g}$. Although our very low temperature data are slightly better explained within $\mathrm{E}_{1 g}$, we remind the reader that $\mathrm{E}_{1 g}$ has difficulties to explain other measurements on $\mathrm{UPt}_{3}$ such as the upper critical field [23] or the Knight shift 40] which both point towards an odd parity OP.

We do not observe any coupling between the low temperature specific heat anomaly and the thermal conductivity at very low temperatures. This points to a localized nature of the modes that lead to this specific heat anomaly. As indicated by Fomin and Flouquet [39] it could be possible that the real magnetic ordering in $\mathrm{UPt}_{3}$ arises only at much lower temperatures $(T \approx 20 \mathrm{mK})$ than indicated by the appearance of a neutron diffraction signal (at $T \approx 5 \mathrm{~K}$ ). This signal could indeed be related to the onset of short range order as a consequence of large magnetic fluctuations, rather than to a real long range magnetic order [39]. But it can not be excluded that a weak interplay between these local moments and the heavy electrons may influence the temperature dependence of $\kappa$ and complicate a quantitative fit.

We have also presented measurements of the overall temperature and field behaviour of the thermal conductivity in the mixed state. The low field low temperature result shows the absence of the expected square root behaviour of the thermal conductivity, for both 
measured axis. We have also seen that the particular field dependence of $\kappa(B)$ near $\mathrm{B}_{c 2}$ is very different along the $\mathrm{b}$ or $\mathrm{c}$ axis axis $\left(d \kappa_{c} / d B \gg d \kappa_{b} / d B\right)$, which may be related to the Pauli limitation of the upper critical field along the c axis. Clearly, both points invite more theoretical calculations and might be deeply connected to the $\mathrm{OP}$ of $\mathrm{UPt}_{3}$. In the intermediate field regime, both the vortex core contribution to $\kappa$ and quasiparticle-vortex scattering are important, and the data are difficult to interpret without further theoretical work. Near $\mathrm{T}_{c}$, the derivative of the thermal conductivity, directly related to the order parameter, shows a clear jump when crossing the $\mathrm{A} \rightarrow \mathrm{C}$ phase boundary. This is a clear signature of the change of symmetry of the OP at this phase transition.

Acknowledgements. We would like to thank very useful discussions with P. Wölfle, P. J. Hirschfeld, A.I. Buzdin and V.P. Mineev. 


\section{REFERENCES}

[1] L.P. Gorkov, Sov. Sci. Rev. A. Phys. Vol. 9, 1-116, 1987; M. Sigrist and K. Ueda, Rev. Mod. Phys. 63, 239 (1991); R. H. Heffner, M. Norman Comments in Cond. Matt. Phys. 17, $361(1996)$.

[2] A. Sulpice, P. Gandit, J. Chaussy, J. Flouquet, D. Jaccard, P. Lejay and J.L. Tholence, J. Low Temp. Phys., 62, 39 (1986).

[3] K. Behnia, L. Taillefer, J. Flouquet, D. Jaccard, K. Maki, Z. Fisk, J. Low Temp. Phys. 84, 261 (1991).

[4] K. Behnia, D. Jaccard, L. Taillefer, J. Flouquet and K. Maki, J. of Mag. and Mag. Mat. 108, 133 (1992).

[5] A.D. Huxley, H. Suderow, J.P. Brison, J. Flouquet, Phys. Lett. A 209 365-372 (1995).

[6] H. Suderow, J. P. Brison, A. Huxley, J. Flouquet, Physica B 223 \& 224, 47-49 (1996).

[7] B. Lussier, B. Ellman, L. Taillefer, Phys. Rev. Lett. 73, 3294 (1994).

[8] B. Lussier, B. Ellman, L. Taillefer, Phys. Rev. B 53, 5145 (1996).

[9] C.J. Pethick and D. Pines, Phys. Rev. Lett. 57, 118 (1986).

[10] S. Schmitt-Rink, K. Miyake and C.M. Varma, Phys. Rev. Lett. 57, 2575 (1986).

[11] H. Monien, K. Scharnberg, L. Tewordt and D. Walker, Solid State Commun. 61, 581 (1987).

[12] B. Arfi and C.J. Pethick Phys. Rev. B 38, 2312 (1988).

[13] P. Hirschfeld, D. Vollhardt and P. Wölfle, Solid State Commun. 59, 111 (1986).

[14] P.J. Hirschfeld, P. Wölfle and D. Einzel Phys. Rev. B, 37,83 (1988).

[15] M. J. Graf, S.K. Yip, J.A. Sauls and D. Rainer Phys. Rev. B 53, 15147 (1996). 
[16] M.J. Graf, S.K. Yip and J.A. Sauls J. Low Temp. Phys. 102, 1 (1996).

[17] A. Fledderjohann et al. Solid State Commun. 94, 163 (1995).

[18] M. R. Norman, P. J. Hirschfeld, Phys. Rev. B, 53, 5706 (1996).

[19] Yu. S. Barash and A.A. Svidzinskii, Phys. Rev. B 53, 15254 (1996).

[20] B.K. Chakalskii, N.A. Redko, S.S. Shalty, V.M. Azhazha, Sov. Phys. JETP 48(4), Oct. 1978.

[21] L. Taillefer, F. Piquemal and J. Flouquet Physica C 153, 451 (1988)

[22] S. Kambe, private communication.

[23] C.H. Choi and J.A. Sauls Phys. Rev. Lett. 66, 484 (1991).

[24] K. Behnia, D. Jaccard, J. Sierro, P. Lejay, J. Flouquet Physica C 196, 57 (1992).

[25] G. Yin, K. Maki, Phys. Rev. B 47, 892 (1993).

[26] G. Yin, K. Maki, Physica B 199,200, 224 (1994).

[27] J. Lowell, J.B. Sousa, J. Low Temp. Phys. 3 (1970).

[28] W.F. Vinen, E.M. Forgan, C.E. Gough and M.J. Hood, Physica 55 (1971).

[29] A.P. Ramirez, N. Stücheli, E. Bucher, Phys. Rev. Lett. 74, 1218 (1995).

[30] G. E. Volovik, JETP Lett., 58, 6 (1993).

[31] P.A. Lee, Phys. Rev. Lett. 71, 1887 (1993).

[32] L.S. Borkowski, P.J. Hirschfeld, W.O. Putikka Phys. Rev. B, 52, R3856 (1995).

[33] G. Preosti, H. Kim, P. Muzikar Phys. Rev. B 50, 13638 (1994).

[34] A.V. Balatsky, M.I. Salkola, A. Rosengren Phys. Rev. B 51, 15547 (1995).

[35] A. Houghton, K. Maki Phys. Rev. B, 4, 843 (1971). 
[36] R. M. Clearly Phys. Rev. B, 1, 169 (1970).

[37] J. P. Brison et al. J. Low Temp. Phys. 95, 145 (1994).

[38] E.A. Schubert, H.B. Strickler and K. Andres, Phys. Rev. Lett. 68,117 (1992).

[39] I.A. Fomin and J. Flouquet Solid State Commun. 98, 795 (1996).

[40] H. Tou, Y. Kitaoka, K. Asayama, N. Kimura, Y. Onuki, E. Yamamoto and K. Maezawa Phys. Rev. Lett. 77, 1374 (1996). 


\section{FIGURES}

FIG. 1. The thermal conductivity of $\mathrm{UPt}_{3}$ below $1 \mathrm{~K}$ for the heat current along the $\mathrm{b}$ and along the $\mathrm{c}$ axis. The zero field data are shown together with the normal phase data, measured at $3 \mathrm{~T}$.

FIG. 2. The anisotropy $\kappa_{c} / \kappa_{b}$ normalized to 1 at $\mathrm{T}_{c}^{-}=480 \mathrm{mK}$ is shown as a function of the reduced temperature $\mathrm{T} / \mathrm{T}_{c}^{-}$. The inset shows a kink of $\kappa_{c} / \kappa_{b}$ at $\mathrm{T}_{c}^{+}=550 \mathrm{mK}$. Note the low error bars compared with earlier work (Refs. [3,5, [, 8]).

FIG. 3. The very low temperature thermal conductivity of $\mathrm{UPt}_{3}$ (between $70 \mathrm{mK}$ and $16 \mathrm{mK}$ ) as a function of $\mathrm{T}^{2}$, with the fits based on the predictions of Graf et al. 15. The inset shows $\kappa / T$ between $34 \mathrm{mK}$ and $16 \mathrm{mK}$. Below $30 \mathrm{mK}$, the properties of the impurity induced band of quasiparticles are observed (see text).

FIG. 4. Some of our temperature scans at constant magnetic fields are shown in this figure. The inset shows the observed anomaly at $\mathrm{T}_{c}$, compared to the resistive transition at $1.56 \mathrm{~T}$.

FIG. 5. Phase diagramm of $\mathrm{UPt}_{3}$ deduced from the thermal conductivity measurements. The $\mathrm{A} \rightarrow \mathrm{N}$ and $\mathrm{C} \rightarrow \mathrm{N}$ transitions are traced by the anomaly on the temperature scans which appear at $\mathrm{T}_{c}$ under magnetic field. The $\mathrm{B} \rightarrow \mathrm{C}$ phase transition is seen on the field dependence of $\kappa$ (see Fig. 8). No clear sign of the $A \rightarrow B$ transition was seen in our measurements. Lines are guides to the eye.

FIG. 6. The slopes obtained with our temperature scans under magnetic fields. Lines are guides to the eye : continuous lines in the A phase, and dashed dotted lines in the $\mathrm{C}$ phase. The error bars, which become more important at high fields due to the broadening of the transition are also shown.

FIG. 7. The figure shows $\kappa / T(B)$ for $\mathrm{T} \rightarrow 0 \mathrm{~K}$ at low fields. Note that surprisingly, $\kappa_{b}$ and $\kappa_{c}$ show a linear behaviour (see text). At very low fields, close to $\mathrm{B}_{c 1}$ a curvature of $\kappa(B)$ is observed, but in this region a non negligible irreversibility exist. 
FIG. 8. The magnetic field dependence of $\kappa / T(B)$ at some representative temperatures together with the zero temperature extrapolation of $\kappa / T(B)$ (open circles). Note that, while $\kappa_{b} / T$ is roughly linear with the magnetic field, $\kappa_{c} / T$ shows a strong upturn near $\mathrm{B}_{c 2}$ up to the normal phase value.

FIG. 9. The magnetic field dependence of the thermal resistivity $W(B)=1 / \kappa(B)$ at low fields normalized to the value at zero field $\mathrm{W}_{0}$. Lines are guides to the eye. Note that our measurements, more extensive than in Refs. [3, [5], show that $W$ is not linear in B at low fields (see text). 
This figure "Fig_1.GIF" is available in "GIF" format from: http://arxiv.org/ps/cond-mat/9702089v1 
This figure "Fig_2.GIF" is available in "GIF" format from: http://arxiv.org/ps/cond-mat/9702089v1 
This figure "Fig_3.GIF" is available in "GIF" format from: http://arxiv.org/ps/cond-mat/9702089v1 
This figure "Fig_4.GIF" is available in "GIF" format from: http://arxiv.org/ps/cond-mat/9702089v1 
This figure "Fig_5.GIF" is available in "GIF" format from: http://arxiv.org/ps/cond-mat/9702089v1 
This figure "Fig_6.GIF" is available in "GIF" format from: http://arxiv.org/ps/cond-mat/9702089v1 
This figure "Fig_7.GIF" is available in "GIF" format from: http://arxiv.org/ps/cond-mat/9702089v1 
This figure "Fig_8.GIF" is available in "GIF" format from: http://arxiv.org/ps/cond-mat/9702089v1 
This figure "Fig_9.GIF" is available in "GIF" format from: http://arxiv.org/ps/cond-mat/9702089v1 